\title{
The Constant Pressure Water Supply System Based on PROFINET Technology
}

\author{
ZHOU Zhi-feng \\ Shanghai Dianji University
}

\begin{abstract}
In the production of steel rolling, Cooling water is important to the quality of steel, This article in view of the continuous steel rolling production of water supply system is put forward higher requirements , In the design of water supply system adopted the PROFINET industrial Ethernet technology and frequency conversion technology. Did the constant pressure, steady flow, fast communication, precise control. In this paper on the selection of PROFINET and expounds in detail the hardware and software configuration.
\end{abstract}

Index Terms - constant pressure water supply Industrial Ethernet PROFINET configuration

\section{Introduction}

With the development of frequency conversion technology and computer control technology, constant pressure water supply is not yet a new topic any more and it has been applied in many industries. However, in the production of large iron \& steel industry, it is hard to realize accuracy control due to large water usage, many influencing factors and low information and communication speed. Thus in the production of iron \& steel, the water system is always controlled independently. Nevertheless, with the continuous improvement of the requirements of product quality on cooling water, the parameters of water have become an important part of the quality model, which not only require the reliable supply of water source, but also conduct accuracy control on the water pressure, water quality, flow and temperature, achieving the stability. The water control accuracy has become one of the important factors affecting the quality of products. Therefore, the collected water system information shall be conveyed to the control system of the main production line accurately, timely and swiftly. In other words, the water supply is no longer an independent control system, but an organic part of the entire production line control system. It is critical to choose the control system and computer network technologies.

Since the 1980s, people have paid attention to the relationships between water and the quality of products and have been hoping to incorporate the water system into the entire control system to meet the swift and accurate requirements. But both the computer centralized control system and distributed control system (DCS) fail to solve this problem. Since the appearance of field bus (FCS) in 1990s, people see the hope and it truly fills in the gaps of DCS, including many structure levels, low communication efficiency, closed communication protocol and unfavorable cross-system connection. It realizes the interconnection of the bottom field devices or field instruments of process automation and the basic automation, and solves the integration issues of field devices, instruments and control system. Proved through practice, the field bus really brings great change to the control system, but it also has the problems like many varieties and incompatibility. Its main structure is still the master-slave structure and could not realize one network to the bottom. Due to compatibility problems, it will be inconvenient in the transformation of the existing production lines. Therefore, through repeated demonstration, we adopt the PROFINET industrial Ethernet technology in the design and the effect is satisfying.

\section{Technical program selection demonstration}

\subsection{Technological requirements}

The original water supply has many faults due to the control technology and equipment aging and the excessive pressure fluctuations. Because the water system is not connected with the control system of main rolling line, the information can not be exchanged, which has seriously affected the improvement of product quality. According to the needs of production and the status of control system, the users have brought forward technological requirements as follows:

1) The pressure of the pipe network beginning end of water system is $3 \mathrm{MPa}$, the pressure of the water supply farthest point is $1.5 \mathrm{MPa}$ and the fluctuation range is less than $15 \%$.

2 ) The start and stop shall be stable and avoid the occurrence of water hammer. The rapidity of start is not required.

3) It shall have good compatibility with the original control system of the main rolling line, the communication between them must be fast and precise and all data shall be sent to the management system of the main rolling line duly.

4 ) The reconstruction shall consider the possibility of the upgrading of control system of the main rolling line and ensure the advance of technology.

5 ) The control room has the functions of remote monitoring, fault diagnosis alarm, production reporting, remote operation, etc. It is easy to operate, economical and practical, and owns friendly human-computer interface.

\subsection{Selection of main circuit program}

The design task is to reconstruct the cooling water system of finishing mill. This system has 4 sets of water pumps, with motor model: Y3-315M, voltage: $380 \mathrm{~V}$ and power: $132 \mathrm{~kW}$. Three motors work, while one is spare. Two sets of the commonly used pumps (M1 and M2) adopt AC power frequency drive, the other commonly used pump (M3) and the 
spare pump (M4) adopt AC variable frequency drive. Due to the accurate calculation on water consumption, under normal circumstances, M3 works within the range of $25-50 \mathrm{~Hz}$ to maintain the pressure stabilization. In exceptional circumstances, if the operating frequency of M3 is less than 10 $\mathrm{Hz}$, it shall turn the M3 into power frequency and M4 is put into a variable frequency drive. Conversely, when the operating frequency of M4 is very low, it shall cut off M4 automatically and turn M3 into variable frequency. Diagram-1 is the power supply system single-line diagram.

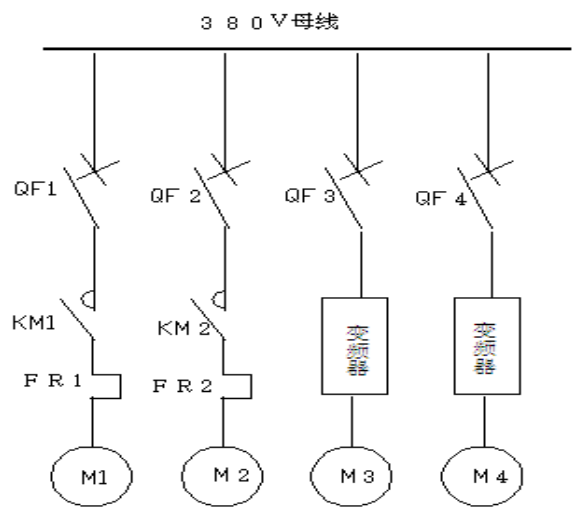

Diagram-1 Power Supply System Single-line Diagram

\subsection{Selection of control technology program}

\subsubsection{Structure of computer control system}

As for the production line with rolling speed of $120 \mathrm{~m} / \mathrm{s}$, the flow and pressure of water are associated with the specifications, rolling speed and steel billet temperature of the rolled products, so the performance of control system is of the utmost importance and is required to have high control accuracy, fast communication and good safety and reliability. We have selected the comparatively mature two-level computer controlled system, namely, the first level is basic automation and mainly focuses on Siemens S7-300PLC; the second level is the process control and mainly focuses on industrial controller and has set up the engineer station and operator station. All information is delivered to the engineer station, operator station and the main rolling line management computer through the industrial Ethernet interface. See diagram-2 for the system structure.

\subsubsection{Selection of industrial Ethernet technology}

The control system of the former main production line is the PROFIBUS bus that was put into operation in 1998, and the water system adopts the distributed control system of Henghe. The reconstruction shall fully consider the compatibility with the control system of the main rolling line. After analyzing the advantages of various networks like PROFIBUS and PROFINET and the busing technology comprehensively, we finally select PROFINET Ethernet technology.

PROFINET, launched by PROFIBUS International (PI), is the new generation of automation bus standard based on the industrial Ethernet technology. It could provide the perfect solutions and technologies of current automation field including real-time Ethernet, motion control, distributed automation, fail safety and network security, and it can be fully compatible with existing field bus (like PROFIBUS) technology. PROFINET is a full open and digitized network and uses the TCP/IP and IT standards. It could realize the seamless connection of industrial control network and enterprise information network. PROFINET technology owns good compatibility and is more suitable for the reconstruction of existing system.

In comparison with PROFIBUS, the structure of PROFINET is simpler and communication efficiency is higher. As we know, the topology of the PROFIBUS bus system is a master-slave structure. One system has many masters, many slaves and the network is divided into two levels, namely PROFIBUS-DP and PROFIBUS-FMS. PROFIBUS-DP is responsible for the communication between the master and slave, while PROFIBUS-FMS is responsible for the communication between the master and mater, mater and host computer, whose communication information has multi-layer exchange and its efficiency is low. PROFEINET adopts the standard Ethernet technology and its topological structure is the same as the commercial Internet. All information is exchanged through the switch and there is one level of network communication. Thus, its exchange efficiency is higher than the bus system. See diagram-2 for the PROFINET system sketch map.

As seen from diagram-2, the converter, PLC and remote $\mathrm{I} / \mathrm{O}$ of the four pumps is connected to the switch, PLC, converter, host computer (engineer station and operator station) through the network, and the communication with the former FROFEIBUS system is connected by the switch.

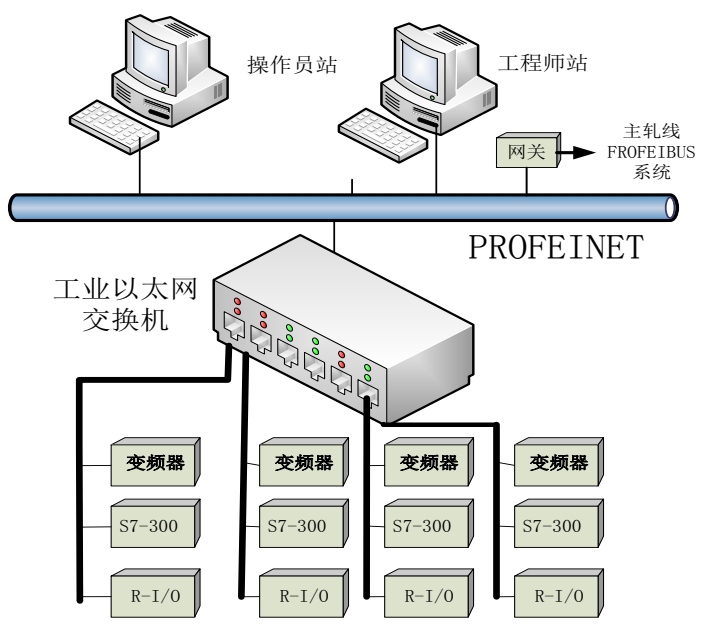

Diagram-2 Computer and PROFINET Structure Sketch Map

\section{Hardware configuration}

\subsection{Network configuration}

PROFINET network topology consists of star structure, linear structure, tree and ring structure. As for water system, it 
is a small system, so the star topology is adopted, which is featured by simple network structure and good expansibility. The entire structure centers on switch and establishes connection among all equipment. There are various interfaces in the switch, which could connect a variety of transmission media; every node and the central node use separate connecting line, thus the fault of single node will not influence the entire network, which is easy to treat the node fault; the cost and maintenance expense are low. The drawback is that the switch is the information exchange center and it can not fail, otherwise, the influence is huge. Therefore, it shall pay special attention to the reliability and applicability when selecting the hardware. We have selected the Siemens branded IPC and switch, which have good performance and nice suitability with PROFINET. Network configuration parameters are as follows:

\section{1) Switch}

Model: SCALANCE XB008G

Parameter: 8 10/100/1000 Mbit/s RJ45 interface, owning adaptive function.

2) Industrial control unit (ICU)

Model: SIMATIC IPC627C

Processor: Intel Core i3-330E (2C/4T 、 $2.13 \mathrm{GHz}$ - HT 、 VT 、 3 MB buffer memory)

Hardware: 250 GB HDD ;

System: Windows XP Professional, Multi-Language

Onboard PROFINET 、 3 x RJ45 、 CP1616- compatible; PROFINET model provides battery backup and onboard 2 MB SRAM

3) PLC hardware configuration

Model: Siemens SIMATIC S7-300

Power supply: PS 307 2A (120/230 VAC : 24 VDC / 2

A)CPU: $315-2$ PN/DP

work memory; $256 \mathrm{~KB}$;

PROFINET IO-Controller; supports RT;

PROFINET interface and 1 port;

PROFINET CBA-Proxy; TCP/IP transport protocol;

Digital module: DI32/DO32x24V/0.5A

Analog input module: SM 321; 8 node;

Analog output module: SM 322; 8 node;

4) Converter:

Model: MICROMASTER 440

Voltage: triphase $400 \mathrm{~V}$

Power: $120 \mathrm{KW}$

Protection level: IP65

This converter adopts the high-performance vector control system and owns good low-speed characteristics and strong overloading capacity. It possesses good human-computer interface and is easy to connect and communicate with PLC. It is suitable to control the high-performance constant pressure water supply system.

\section{WINCC software configuration}

As above mentioned, this system adopts two-level computer system. The host computers are engineer station and operator station. Their functions are fault diagnosis, data reporting, operation monitoring, model parameters modifying and remote control. The host computer is required to own good human-computer interface (HMI). Considering from many angles, we select the Siemens WCC6.0 configuration software. WCC software is a HMI/SCADA software developed by Siemens AG on the win 2000 and windows XP platforms of Microsoft. It possesses good openness and flexibility and can meet the control requirements of the production and process automation of the project. WCC includes many editors like graphic design, global script, variables archiving, alarming, reporting and user management project safety, and it owns a high degree of practicality and convenience.

When designing the screen, the working status of all equipment is displayed in the screen in the form of graph and is distinguished by different colors or flashings.

When programming the control screen, failure screen and monitor screen, it could be finished by using the modules in the graphics library, which is very convenient. Diagram-3 is a simple operation screen. It could make the water pump operation by assigning a value to the button or signal lamp in the programme.

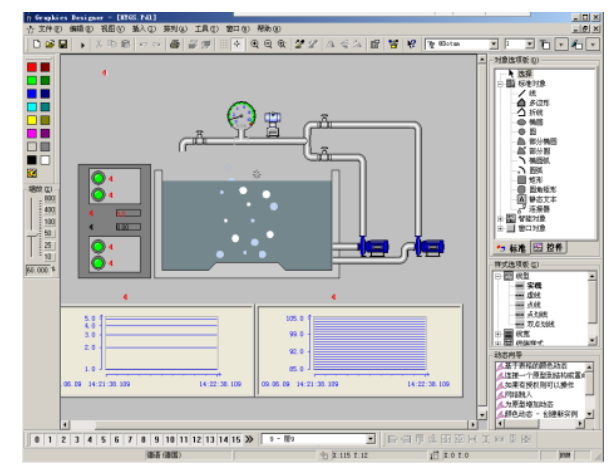

Diagram-3 Monitor Screen Switching

Remote monitor is one of the main functions of host computer. It could monitor the value, curve or graph. All screens could be switched. When designing, it only needs to fill corresponding data into the table. Diagram-4 is the pressure value assignment screen design. 


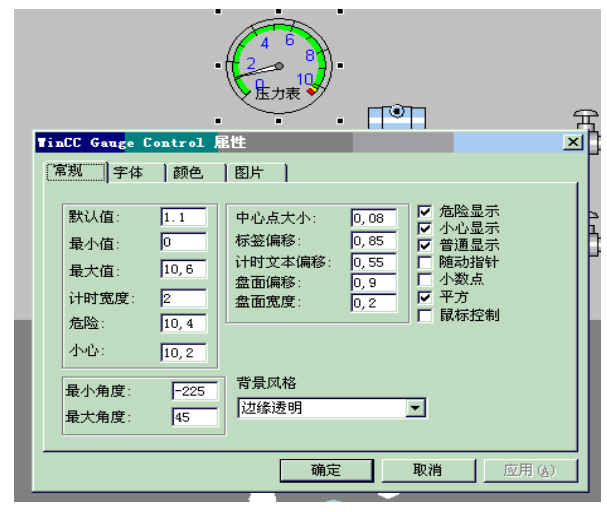

Diagram-4 Pressure Monitoring Screen

\section{Conclusions}

Through two years' operation, the system operates stably, control accuracy is high and operation is convenient. It achieves seamless connection with the former system and lays foundation for the upgrading of the former system in the future. The reconstruction meets the technical requirements and reaches the desired purpose, as well as provides experience and support for the technical reconstruction of other industries.

\section{References:}

[1] $\mathrm{Fu}$ Lei, Ma Buqiang, Continuous Casting Automation System Design Based on PROFINET Industrial Ethernet [J].Heavy Machinery.2012 , 2.41-43.

[2] Zhang Dandan, Overview of Industrial Network PROFINET Based on Ethernet.Manufacturing Automation.2011 , 2 , 2 , (33) .198-203.

[3] Lou Xiaohong. 1200mm Cold Rolling Mill Column Electric Drive Control System Automation Technological Transformation[J].Electric Drive.2009 , 8 , 4.41-45.

[4] Cui Jian. Siemens Industrial Network Communications Guide [M].Beijing, Machinery Industry Press.2004.9 